\title{
Tetragonal modular curves
}

\author{
by \\ Daeyeol Jeon and Euisung Park (Seoul)
}

0. Introduction. A smooth projective curve $X$ defined over an algebraically closed field $k$ is called $d$-gonal if it admits a map $\phi: X \rightarrow \mathbb{P}^{1}$ over $k$ of degree $d$. If the genus $g \geq 2$ and $d=2$ then $X$ is called hyperelliptic. We will say that $X$ is trigonal, tetragonal and pentagonal for $d=3, d=4$ and $d=5$ respectively.

Let $N$ be a positive integer, and let

$$
\Gamma_{0}(N):=\left\{\left(\begin{array}{ll}
a & b \\
c & d
\end{array}\right) \in \mathrm{SL}_{2}(\mathbb{Z}) \mid c \equiv 0 \bmod N\right\} .
$$

Let $X_{0}(N)$ denote the modular curve corresponding to $\Gamma_{0}(N)$. Then Zograf [Z] gave a linear bound on the level $N$ of $d$-gonal modular curves $X_{0}(N)$. Also Nguyen and Saito [N-Sa] proved an analogue of the strong Uniform Boundedness Conjecture for elliptic curves defined over function fields of dimension one by using the connection with giving a bound on the level $N$ of $d$-gonal modular curves $X_{0}(N)$.

Recently, Hasegawa and Shimura $[\mathrm{H}-\mathrm{S}]$ gave a highly sharpened upper bound for $3 \leq d \leq 5$ by trying to determine $d$-gonal modular curves $X_{0}(N)$ for such $d$. For $d=2$ it was done by Ogg [O]. Actually Hasegawa and Shimura succeeded in determining all trigonal modular curves $X_{0}(N)$ but failed for tetragonal and pentagonal $X_{0}(N)$.

The following lists $N$ for which they did not know whether $X_{0}(N)$ was tetragonal or not:

$76,82,84,88,90,93,97,99,106,108,109,113,115,128,133,137$, $157,169$.

In this work we prove that $X_{0}(N)$ is tetragonal only for $N=88,99,109$ among the above 18 numbers. Combining with the result of $[\mathrm{H}-\mathrm{S}]$ we get the following:

2000 Mathematics Subject Classification: 11G18, 11G30.

Key words and phrases: modular curve, tetragonal. 
Theorem 0.1. The modular curve $X_{0}(N)$ has $\operatorname{Gon}\left(X_{0}(N)\right)=4$ if and only if

$$
\begin{aligned}
g=5: & N=42,51,52,55,56,57,63,65,67,72,73,75 \\
g=6: & N=58,79,121 \\
g=7: & N=60,62,68,69,77,80,83,85,89,91,98,100 \\
g=8: & N=74,101,103,109,125 \\
g=9: & N=66,70,87,88,95,96,99,107 \\
g=10: & N=92 \\
g=11: & N=78,94,104,111,119,131 \\
g=13: & N=143 \\
g=14: & N=167 \\
g=16: & N=191 \\
g=17: & N=142 .
\end{aligned}
$$

REMARK 0.2. Note that rational, elliptic and hyperelliptic curves always admit tetragonal maps. One can find a list of those curves $X_{0}(N)$ in $[\mathrm{O}]$. For $d \geq \frac{1}{2} g+1$, any curve of genus $g$ has a $d$-gonal map [K-L]. Since all the modular curves $X_{0}(N)$ with $\operatorname{Gon}\left(X_{0}(N)\right)=3$ are of genus $g=3,4$ (see $[\mathrm{H}-\mathrm{S}]$ ), they are tetragonal. Combining with Theorem 0.1 one can get a complete list of $X_{0}(N)$ which are tetragonal.

1. Definitions and general results. Let $X$ be a smooth projective curve of genus $g \geq 4$.

1.1. Gonality. For a line bundle $L \in \operatorname{Pic} X$, a subspace $V \subset H^{0}(X, L)$ is said to be a $g_{d}^{r}$ if $\operatorname{deg}(L)=d$ and $\operatorname{dim}(V)=r+1$. The gonality of $X$ is

$$
\operatorname{Gon}(X):=\min \left\{d \mid X \text { has a } g_{d}^{1}\right\} .
$$

In this paper, when we say that $X$ is $d$-gonal it does not mean $\operatorname{Gon}(X)=d$ but just that $X$ admits a $g_{d}^{1}$. Thus $d$-gonal curves $X$ may have $\operatorname{Gon}(X)<d$. For example all hyperelliptic curves are automatically tetragonal.

1.2. Clifford index. For a line bundle $L \in \operatorname{Pic} X$, the Clifford index of $L$ is the integer

$$
\operatorname{Cliff}(L):=\operatorname{deg}(L)-2\left(h^{0}(X, L)-1\right)
$$

and the Clifford index of $X$ itself is defined as

$$
\operatorname{Cliff}(X):=\min \left\{\operatorname{Cliff}(L) \mid h^{0}(X, L) \geq 2, h^{1}(X, L) \geq 2\right\} .
$$

It is well known that $\operatorname{Cliff}(X)+2 \leq \operatorname{Gon}(X) \leq \operatorname{Cliff}(X)+3$ (see $[\mathrm{C}-\mathrm{M}]$ ).

1.3. Clifford dimension. We will say that $L \in \operatorname{Pic} X$ contributes to the Clifford index of $X$ if both $h^{0}(X, L) \geq 2$, and $h^{1}(X, L) \geq 2$, and that $L$ 
computes the Clifford index of $X$ if in addition $\operatorname{Cliff}(X)=\operatorname{Cliff}(L)$. The Clifford dimension of $X$ is defined as

$$
\min \left\{h^{0}(X, L)-1 \mid L \text { computes the Clifford index of } X\right\} .
$$

If a line bundle $L$ achieves the minimum and computes the Clifford index, then we will say that $L$ computes the Clifford dimension. In most cases, the Clifford dimension $\ell$ is equal to 1 and the curves with $\ell \geq 2$ are rather rare. It is a classical result that $\ell=2$ if and only if $X$ is a smooth plane curve of degree $\geq 5$. The case $\ell=3$ was settled by Martens $[M]$. He proved that $\ell=3$ if and only if $X$ is a complete intersection of two irreducible cubic surfaces in $\mathbb{P}^{3}$, and hence its genus $g$ is 10 .

1.4. Property $N_{p}$. If $X$ is a non-hyperelliptic curve, then the canonical line bundle $\omega_{X}$ defines an embedding $X \hookrightarrow \mathbb{P} H^{0}\left(X, \omega_{X}\right)=\mathbb{P}^{g-1}$. Consider the minimal free resolution

$$
0 \rightarrow F_{g-2} \rightarrow \cdots \rightarrow F_{2} \rightarrow F_{1} \rightarrow S \rightarrow S_{X} \rightarrow 0
$$

of the homogeneous coordinate ring $S_{X}=S / I_{X}$ as an $S$-module where $S=\mathbb{C}\left[X_{0}, X_{1}, \ldots, X_{g-1}\right]$ and $F_{i}=\bigoplus_{j \in \mathbb{Z}} S(-i-j)^{\beta_{i, j}}$. We call $\beta_{i, j}$ the graded Betti numbers. Due to Green and Lazarsfeld [G-L], $X \hookrightarrow \mathbb{P}^{g-1}$ is said to have property $N_{p}$ if the resolution is of the form

$$
\cdots \rightarrow S^{\beta_{p, 1}}(-p-1) \rightarrow \cdots \rightarrow S^{\beta_{2,1}}(-3) \rightarrow S^{\beta_{1,1}}(-2) \rightarrow S \rightarrow S_{X} \rightarrow 0 .
$$

Therefore property $N_{1}$ holds if and only if the homogeneous ideal is generated by quadrics, and property $N_{p}$ holds for $p \geq 2$ if and only if it has property $N_{1}$ and the $k$ th syzygies among the quadrics are generated by linear syzygies for all $1 \leq k \leq p-1$. Now we recall the following:

Theorem 1.1 (M. Green and R. Lazarsfeld, Appendix in [G]). Let $X$ be a smooth non-hyperelliptic curve of genus $g \geq 3$. Then the canonical embedding $X \hookrightarrow \mathbb{P}^{g-1}$ fails to have property $N_{p}$ for $p \geq \operatorname{Cliff}(X)$.

Thus if the canonical embedding $X \hookrightarrow \mathbb{P}^{g-1}$ has property $N_{p}$, then $\operatorname{Cliff}(X) \geq p+1$ and $\operatorname{Gon}(X) \geq p+3$.

1.5. Betti numbers. When $\operatorname{Cliff}(X) \leq 2$, Schreyer clarified the relation between the minimal free resolution of the canonical embedding $X \hookrightarrow \mathbb{P}^{g-1}$ and the existence of special linear series of divisors on $X$. For $g \leq 7$, see Table 1 in [Sch1]. For $g \geq 8$, the graded Betti number $\beta_{2,2}$ has one of the values given in the following table [Sch2]:

\begin{tabular}{ccccc}
\hline$\beta_{2,2}$ & $(g-4)(g-2)$ & $\left(\begin{array}{c}g-2 \\
2\end{array}\right)-1$ & $g-4$ & 0 \\
Linear series & $\exists g_{3}^{1}$ & $\exists g_{6}^{2}$ or $g_{8}^{3}$ & $\exists$ a single $g_{4}^{1}$ & Cliff $(C) \geq 3$ \\
\hline
\end{tabular}


2. Proof. We proceed to prove that $X_{0}(N)$ is tetragonal only for $N=$ $88,99,109$ among the 18 numbers $N$ in $\S 0$. For this purpose we compute the graded Betti numbers of the canonical embedding of $X_{0}(N)$. We use the computer programs "Maple" and "Singular". First we calculate the homogeneous ideal of the canonical embedding of $X_{0}(N)$ by using Maple.

Note that, for such $N, X_{0}(N)$ is not hyperelliptic [O]. Thus $X_{0}(N)$ can be identified with the canonical curve which is the image of the canonical embedding

$$
X_{0}(N) \ni P \mapsto\left(f_{1}(P): \cdots: f_{g}(P)\right) \in \mathbb{P}^{g-1}
$$

where $\left\{f_{1}, \ldots, f_{g}\right\}$ is a basis of the space of cusp forms of weight 2 on $X_{0}(N)$. One can get such a basis and the corresponding Fourier coefficients from [St]. Then to obtain the minimal generating system of the homogeneous ideal $I\left(X_{0}(N)\right)$, we only have to compute the relations of the $f_{i} f_{j}(1 \leq i, j \leq g)$ by Petri's theorem. Since there are $(g-2)(g-3) / 2$ linear relations among the $f_{i} f_{j}$, we get quadric generators $Q_{k}\left(x_{1}, \ldots, x_{g}\right)$ with $1 \leq k \leq(g-2)(g-3) / 2$ by assigning $x_{i}$ to $f_{i}$ (for details see [H-S]).

Now we compute the Betti numbers by using Singular. In fact when the genus of $X_{0}(N)$ is big then Singular does not work efficiently. Note that since the canonical embedding is always projectively Cohen-Macaulay, the Betti numbers of the canonical curve are equal to those of the hyperplane section, which allows us to get Betti numbers easier.

We exhibit the so-called Betti table of the canonical embedding for our cases in Table 1.

CASE 1. The canonical embeddings of $X_{0}(N)$ for

$$
N=76,82,84,90,93,97,106,108,113,115,128,133,137,157,169
$$

have property $N_{p}$ for $p \geq 2$. Thus $\operatorname{Gon}\left(X_{0}(N)\right) \geq 5$ by $\S 1.4$.

CAse 2. The curve $X_{0}(88)$ is of genus 9 and $\beta_{2,2}=9-4=5$. Thus it is tetragonal by $\S 1.5$.

Case 3. For $N=99$ and 109, Cliff $\left(X_{0}(N)\right)=2$. Indeed, by $\S 1.4$, Cliff $\left(X_{0}(N)\right) \geq 2$ because the canonical embeddings of these curves have property $N_{1}$. Also $\operatorname{Cliff}\left(X_{0}(N)\right) \leq 2$ since they have a $g_{6}^{2}$ or a $g_{8}^{3}$ by $\S 1.4$. Therefore Cliff $\left(X_{0}(N)\right)$ is computed by a $g_{2+2 \ell}^{\ell}$ where $\ell$ denotes the Clifford dimension. It remains to show that $\ell=1$. We already know that $\ell \leq 3$ from the existence of a $g_{6}^{2}$ or a $g_{8}^{3}$. Since a smooth plane curve of degree $d$ is of genus $(d-1)(d-2) / 2$, the curves $X_{0}(99)$ and $X_{0}(109)$ cannot be plane curves. Thus $\ell \neq 2$ by $\S 1$.3. Also $\ell \neq 3$ because their genera are less than 10 . Therefore Cliff $\left(X_{0}(N)\right)$ is computed by a $g_{4}^{1}$ and hence $X_{0}(99)$ and $X_{0}(109)$ are tetragonal. 
Table 1. The graded Betti numbers for the canonical embedding

\begin{tabular}{|c|c|c|c|c|c|c|c|c|c|}
\hline \multirow[t]{2}{*}{ Genus } & \multirow[t]{2}{*}{$X_{0}(N)$} & $\beta_{1,2}$ & $\beta_{2,2}$ & $\beta_{3,2}$ & \multirow[t]{2}{*}{ Genus } & \multirow[t]{2}{*}{$X_{0}(N)$} & $\beta_{1,2}$ & $\beta_{2,2}$ & $\beta_{3,2}$ \\
\hline & & $\beta_{1,1}$ & $\beta_{2,1}$ & $\beta_{3,1}$ & & & $\beta_{1,1}$ & $\beta_{2,1}$ & $\beta_{3,1}$ \\
\hline \multirow[t]{2}{*}{7} & \multirow[t]{2}{*}{$X_{0}(97)$} & 0 & 0 & 16 & \multirow[t]{2}{*}{9} & \multirow[t]{2}{*}{$X_{0}(128)$} & 0 & 0 & 0 \\
\hline & & 10 & 16 & 0 & & & 21 & 64 & 70 \\
\hline \multirow[t]{6}{*}{8} & \multirow[t]{2}{*}{$X_{0}(76)$} & 0 & 0 & 21 & \multirow[t]{2}{*}{10} & \multirow[t]{2}{*}{$X_{0}(108)$} & 0 & 0 & 20 \\
\hline & & 15 & 35 & 21 & & & 28 & 105 & 162 \\
\hline & \multirow[t]{2}{*}{$X_{0}(109)$} & 0 & 14 & 35 & \multirow[t]{10}{*}{11} & \multirow[t]{2}{*}{$X_{0}(84)$} & 0 & 0 & 0 \\
\hline & & 15 & 35 & 35 & & & 36 & 160 & 315 \\
\hline & \multirow[t]{2}{*}{$X_{0}(169)$} & 0 & 0 & 21 & & \multirow[t]{2}{*}{$X_{0}(90)$} & 0 & 0 & 0 \\
\hline & & 15 & 35 & 21 & & & 36 & 160 & 315 \\
\hline \multirow[t]{10}{*}{9} & \multirow{2}{*}{$X_{0}(82)$} & 0 & 0 & 8 & & \multirow{2}{*}{$X_{0}(115)$} & 0 & 0 & 0 \\
\hline & & 21 & 64 & 70 & & & 36 & 160 & 315 \\
\hline & \multirow[t]{2}{*}{$X_{0}(88)$} & 0 & 5 & 24 & & \multirow[t]{2}{*}{$X_{0}(133)$} & 0 & 0 & 0 \\
\hline & & 21 & 64 & 75 & & & 36 & 160 & 315 \\
\hline & \multirow[t]{2}{*}{$X_{0}(93)$} & 0 & 0 & 0 & & \multirow[t]{2}{*}{$X_{0}(137)$} & 0 & 0 & 0 \\
\hline & & 21 & 64 & 70 & & & 36 & 160 & 315 \\
\hline & \multirow[t]{2}{*}{$X_{0}(99)$} & 0 & 20 & 64 & \multirow[t]{4}{*}{12} & \multirow[t]{2}{*}{$X_{0}(106)$} & 0 & 0 & 0 \\
\hline & & 21 & 64 & 90 & & & 45 & 231 & 550 \\
\hline & \multirow[t]{2}{*}{$X_{0}(113)$} & 0 & 0 & 8 & & \multirow[t]{2}{*}{$X_{0}(157)$} & 0 & 0 & 0 \\
\hline & & 21 & 64 & 70 & & & 45 & 231 & 550 \\
\hline
\end{tabular}

\section{References}

[C-M] M. Coppens and G. Martens, Secant spaces and Clifford's theorem, Compos. Math. 78 (1991), 193-212.

[G] M. Green, Koszul cohomology and the geometry of projective varieties I, J. Differential Geom. 19 (1984), 125-171.

[G-L] M. Green and R. Lazarsfeld, Some results on the syzygies of finite sets and algebraic curves, Compos. Math. 67 (1988), 301-314.

[H-S] Y. Hasegawa and M. Shimura, Trigonal modular curves, Acta Arith. 88 (1999), 129-140.

[K-L] S. L. Kleiman and D. Laksov, Another proof of the existence of special divisors, Acta Math. 132 (1974), 163-176.

[M] G. Martens, Über den Clifford-Index algebraischer Kurven, J. Reine Angew. Math. 336 (1982), 83-90.

[N-Sa] K. V. Nguyen and M.-H. Saito, d-gonality of modular curves and bounding torsions, arXiv:alg-geom/9603024 v1.

[O] A. P. Ogg, Hyperelliptic modular curves, Bull. Soc. Math. France 102 (1974), 449-462.

[Sch1] F.-O. Schreyer, Syzygies of canonical curves and special linear series, Math. Ann. 275 (1986), 105-137. 
[Sch2] F.-O. Schreyer, A standard basis approach to the syzygies of canonical curves, J. Reine Angew. Math. 421 (1991), 83-123.

[St] W. A. Stein, http://modular.fas.harvard.edu.

[Z] P. G. Zograf, Small eigenvalues of automorphic Laplacians in spaces of cusp forms, in: Automorphic Functions and Number Theory, II, Zap. Nauchn. Sem. Leningrad. Otdel. Mat. Inst. Steklov. (LOMI) 134 (1984), 157-168 (in Russian).

Korea Institute for Advanced Study (KIAS)

207-43 Cheongnyangni 2-dong

Dongdaemun-gu

Seoul, 130-722 Korea

E-mail: dyjeon@kias.re.kr

puserdos@kias.re.kr

Received on 6.4.2005

and in revised form on 25.7.2005 